\title{
Direct measurement of the quality factor in a two-dimensional photonic-crystal microcavity
}

\author{
S. Y. Lin and Edmond Chow \\ Sandia National Laboratories, P.O. Box 5800, Albuquerque, New Mexico 87185 \\ S. G. Johnson and J. D. Joannopoulos \\ Department of Physics, Massachusetts Institute of Technology, Cambridge, Massachusetts 02139
}

Received July 3, 2001

\begin{abstract}
A new microcavity design is proposed and structures are realized with a two-dimensional photonic-crystal slab. The cavity consists of seven defect holes that encompass a hexagon and is designed to reduce vertical light leakage. From a direct transmission measurement, a $Q$ value of $816 \pm 30$ is achieved at $\lambda=1.55 \mu \mathrm{m}$. This high- $Q$ cavity will permit the realistic realization of spontaneous-emission modification and on-off optical switches. (C) 2001 Optical Society of America

OCIS codes: $140.3580,190.4400,230.3990$.
\end{abstract}

The realization of a high- $Q$ microcavity at optical wavelength $\lambda$ has many important technological consequences. According to the Purcell effect, ${ }^{1}$ the rate of spontaneous emission can be greatly altered inside a high- $Q$ cavity. ${ }^{2-4}$ A high- $Q$ cavity also operates as a bandpass filter for on-off optical switching. ${ }^{5}$ A photonic crystal, a periodically arranged dielectric sub- $\lambda$ structure, offers a unique optical environment for creating such a cavity. Its ability to confine light strongly will lead to the creation of high- $Q$ cavities at optical $\lambda$ and with a small size $\left(\sim \lambda^{3}\right)$. Moreover, its sub- $\lambda$ property permits great geometrical flexibility in designing microcavities. By using different types and combinations of local defects, one can vary both a cavity's resonant frequency and its mode symmetry. ${ }^{4}$

Whereas a three-dimensional (3D) photonic crystal is ideal for creating high- $Q$ cavities, ${ }^{6,7}$ one- and twodimensional (1D and 2D) photonic crystals have the advantage of simplicity of fabrication. ${ }^{8-12}$ Recently Foresi et al. obtained a microcavity with $Q=265$ by using a four-period $(N=4)$ one-dimensional photonic-crystal. $^{13}$ For 2D photonic-crystal cavities there are several reports of analyses of $Q$ factors, mostly estimated from top-emitting photoluminescence data. ${ }^{11,12,14}$ Labilloy et al., however, used guided luminescence to probe a horizontal cavity and obtain a $Q$ value of 200 for $N=9 .{ }^{10}$ A top-scattering geometry was also suggested for add-drop-filter applications. ${ }^{15}$ Although top emission is a result of leakage of light in the vertical direction, which limits cavity $Q$, direct transmission measurements of $2 \mathrm{D}$ crystal cavities at optical $\lambda$ have not been reported, mainly because of the difficulty of precise lateral waveguide coupling. In this Letter we report direct measurement of $Q$ factors of $2 \mathrm{D}$ photonic-crystal microcavities at $\lambda \sim 1.55 \mu \mathrm{m}$. When a new cavity design is used that minimizes vertical leakage, cavity $Q$ is shown to increase exponentially with $N$, and $Q=814$ is achieved for $N=4$.

Our photonic crystal consists of a $2 \mathrm{D}$ triangular array of holes etched through a GaAs slab. The 2D hole array has a lattice constant $a=440 \mathrm{~nm}$. The hole diameter is $d=0.6 a=264 \mathrm{~nm}$ and the etched depth is $\sim 0.6 \mu \mathrm{m}$. The GaAs slab is $220 \mathrm{~nm}$ thick $(t=0.5 a)$ and is sandwiched between a $2-\mu \mathrm{m}$-thick $\mathrm{Al}_{x} \mathrm{O}_{y}$ layer and a $0.1-\mu \mathrm{m} \mathrm{SiO}_{2}$ layer. Previous measurements have shown that the photonic crystal has a large TE-like photonic bandgap, i.e., $0.255<\omega(a / \lambda)<$ $0.325{ }^{16}$ Within the gap, light is guided inside and near the proximity of the GaAs slab through index guiding. ${ }^{16-18}$ The field thus decays exponentially in the air and the substrate, and vertical radiation losses are possible only when translation symmetry is broken, e.g., by a defect cavity.

Our microcavity consists of seven smaller holes, with hole diameters $d^{\prime}=0.4 a=176 \mathrm{~nm}$, that encompass a hexagon and is called a super defect. A scanningelectron microscope image of the $2 \mathrm{D}$ hole array and microcavity is shown in Fig. 1. One may also introduce a cavity by changing the radius of a single hole; however, our calculation predicts that its $Q$ factor will be low. In this case, radiation $Q,\left(Q_{r}\right)$, which describes the rate of decay of the cavity mode into the air, is always less than 500 and usually less than $250 .{ }^{19}$ The superdefect design, however, is expected to have a much

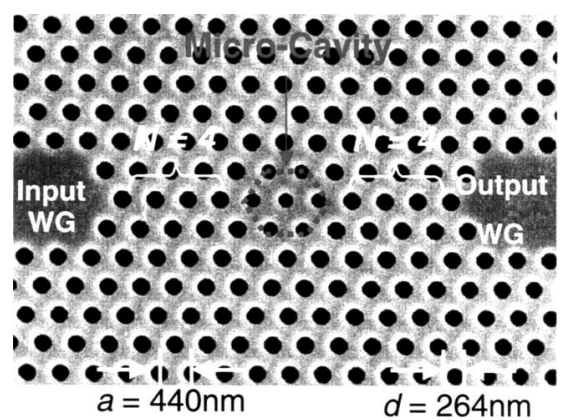

Fig. 1. Scanning-electron microscope top-view image of a microcavity sample. The microcavity consists of seven smaller holes, with hole diameters $d^{\prime}=0.4 a=176 \mathrm{~nm}$, that encompass a hexagon. The input and output waveguides are used to facilitate coupling of laser light. The four periods $(N=4)$ of a photonic crystal act as a photonic tunnel barrier for confinement of light. 
higher $Q_{r},\left(Q_{r}>1000-2000\right)$, and its resonant frequency is near the middle of the TE gap (midgap). One drawback of this design is that the cavity size is slightly increased to $\sim 0.1 \mu \mathrm{m}^{3}$ [or a volume of $1.5 \times(\lambda / n)^{3}$ ], with the cavity mode slightly delocalized and doubly degenerate. Here $n$ is the refractive index of GaAs.

Another loss mechanism is decay of the cavity mode into adjacent waveguides and is described by $Q_{w}$. As shown in Fig. 1, the cavity is separated from the input and output waveguides by four periods, $N=4$, of photonic crystal. The photonic crystal acts as a photon tunnel barrier for coupling light from the input guide to the cavity and then decays back to the output guide. The decay rate, and therefore $Q_{w}$, has an exponential dependence on the thickness of the tunnel barrier, i.e., $N a_{0} \cdot{ }^{20}$ The total $Q_{t}$ is given by $1 /\left(Q_{t}\right)=$ $1 /\left(Q_{w}\right)+1 /\left(Q_{r}\right)$. As one can, in principle, increase $Q_{w}$ by increasing $N, Q_{r}$ sets the upper limit for $Q_{t}$. To realize a high- $Q$ cavity, one must have a cavity design that maximizes $Q_{r}$. It is equally important to demonstrate that $Q_{w}$ depends on $N$ exponentially. ${ }^{21} \quad$ For this experiment, three nominally identical cavities with different values of $N, N=2,3,4$, are fabricated upon the same chip.

A high-resolution tunable diode laser with $0.02-\mathrm{nm}$ scanning steps produces the transmission spectrum. The laser beam is linearly polarized and focused into an input waveguide by a high-numerical-aperture microscope objective. The output light is split and fed into an InGaAs photodetector for intensity measurement and into an infrared camera for mode profile monitoring. This procedure ensures that only the guiding mode signal is fed into the detector.

Figure 2(a) shows a TE transmission spectrum (filled circles) taken from an $N=2$ supercavity sample. The data have a vertical offset of 0.1 for clarity. The spectrum shows a transmission peak at $\lambda \sim 1547 \mathrm{~nm}$; equivalently, $\omega(a / \lambda)=0.2846$, which is at the TE midgap. It also contains multiple-wavelength oscillations, with a periodicity of $\Delta \lambda \sim 0.55 \mathrm{~nm}$. This value of $\Delta \lambda$ corresponds to a resonant cavity of length $L \sim 0.6 \mathrm{~mm}$, which is the distance between the waveguide's end facet and waveguide-crystal interface. The oscillations are thus attributed to Fabry-Perot resonance of the end facets and are not derived from microcavity resonance.

The same measurement is repeated for an $N=4$ cavity sample and the transmission spectrum (filled circles) shown in Fig. 3(a). The data have a vertical offset of 0.08 for clarity. The spectrum shows a better-defined transmission peak as well as shortperiod oscillations. The oscillations have the same period $(\Delta \lambda \sim 0.55 \mathrm{~nm})$ as that for the $N=2$ sample and are due to Fabry-Perot resonance of the end facets. Yet the main transmission peak exhibits several distinct features: First, its position shifts slightly to $\lambda \sim 1551 \mathrm{~nm}, \omega(a / \lambda)=0.2836$, but is still near the TE midgap. Second, its linewidth becomes much narrower; its line shape, more symmetrical and Lorentzian-like. Third, background transmission away from the peak, i.e., $\lambda<1545$ and $\lambda>1555 \mathrm{~nm}$, is near zero. The linewidth narrowing, the improve- ment in line shape as $N$ is increased, and the fact that the peak position is within the TE gap all suggest that the observed transmission peak originates from cavity resonance.

Further transmission measurements, with TMpolarized light, were carried out for the same samples. As there is no TM gap in this wavelength range $(\lambda=1510-1590 \mathrm{~nm})$, no transmission peak is observed. Nonetheless, the Fabry-Perot oscillations persist for TM polarization. This measurement further confirms that the observed peak is due to the presence of a TE photonic bandgap.

To obtain a more-accurate $Q$ value, we first perform a Fourier transform for the raw data and remove the part of Fourier component that is due to Fabry-Perot

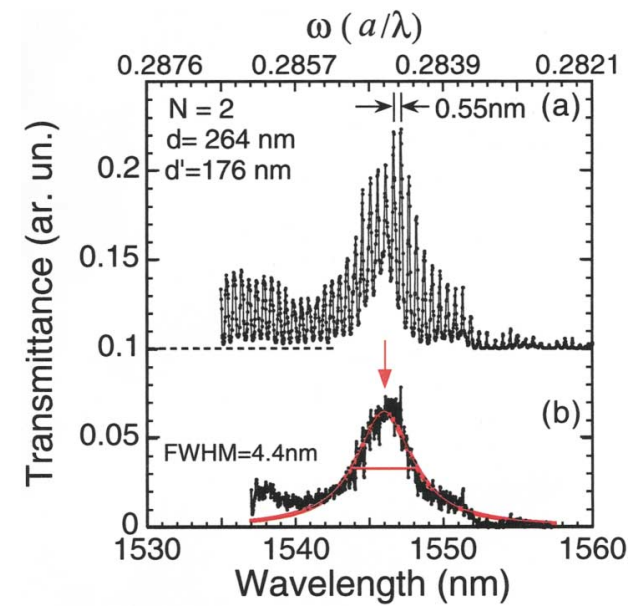

Fig. 2. Transmission spectrum for an $N=2$ microcavity sample. (a) The spectrum, shifted vertically by 0.1 for clarity, shows a transmission peak at $\lambda \sim 1547 \mathrm{~nm}$, or, equivalently $\omega(a / \lambda)=0.2846$. It also contains multiple-wavelength oscillations, with a periodicity of $\Delta \lambda \sim 0.55 \mathrm{~nm}$. (b) Smoothed data (filled circles) fitted to a Lorentzian (red curve); the deduced FWHM is $4.4 \mathrm{~nm}$. ar. un., arbitrary units.

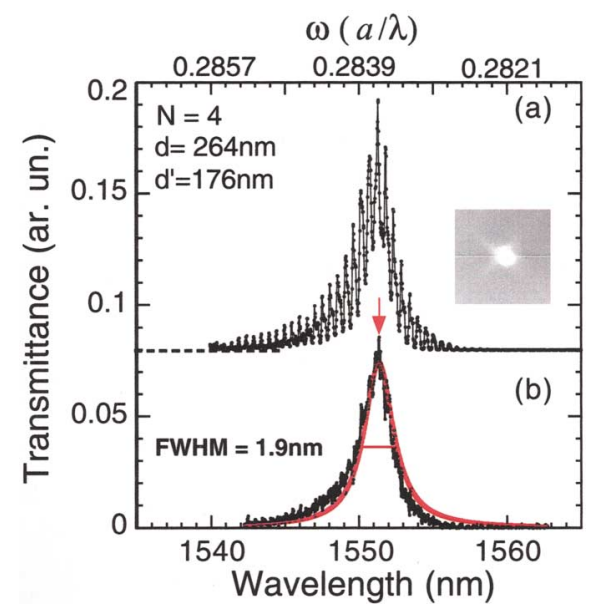

Fig. 3. Transmission spectrum for an $N=4$ microcavity sample. (a) The spectrum, shifted vertically by 0.08 , shows a well-defined transmission peak and also shortperiod oscillations $\Delta \lambda \sim 0.55 \mathrm{~nm}$. (b) The fitted curve (red curve) has a FWHM of $1.9 \mathrm{~nm}$, and the corresponding cavity $Q$ value is $816 \pm 30$. Inset, the infrared image of transmitted light has a well-defined Gaussian-like profile. 


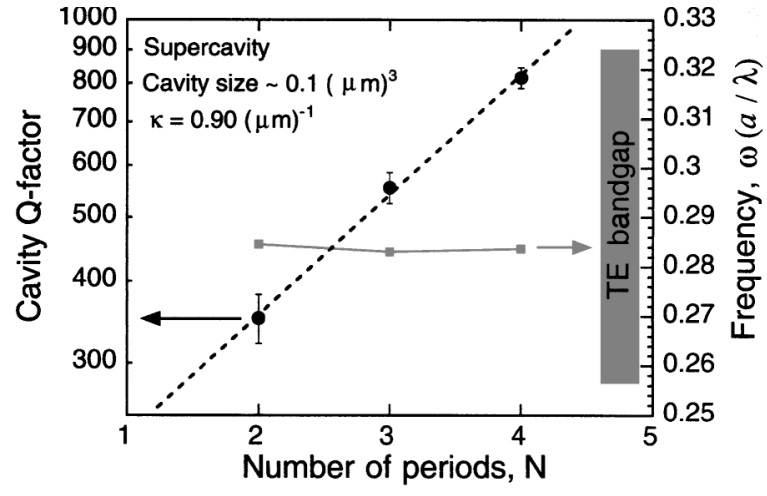

Fig. 4. Summary of resonant frequency (filled squares) and cavity $Q$ factors (filled circles) as a function of $N$. The observed resonant frequency $(\omega)$ is at the TE midgap and varies less than $1 \%$ for all samples. The cavity $Q$ factors are fitted to an exponential function (dashed line), and the deduced slope is $\kappa=0.9(\mu \mathrm{m})^{-1}$.

oscillations. We then apply an inverse Fourier transform to obtain the data, which are plotted as circles in Figs. 2(b) and 3(b). The data are then fitted to a Lorentzian function, and the red curves in Figs. 2(b) and 3(b) represent the fit. The experimental $Q$ factor is given by $Q_{t}=\lambda / \Delta \lambda_{\text {FWHM }}$, where $\lambda$ is the peak wavelength and $\Delta \lambda_{\text {FWHM }}$ is the FWHM. For $N=2$, the experimental $\omega$ and $Q$ values are $\omega=0.2846$ and $Q_{t}=351 \pm 20$, respectively. For $N=4, \omega=0.2837$ and $Q_{t}=816 \pm 30$, which are three times higher than that for the 1D crystal microcavity of $N=4 .^{13}$ This result is expected, as light in a $1 \mathrm{D}$ cavity leaks both vertically and laterally. The measured $\omega$ also agrees with theoretical value $\omega=0.2933$ within $4 \%$. For our $N=4$ cavity, a large spontaneous-emission enhancement rate, $\eta \sim Q /\left(\Delta V / \lambda^{3}\right)=514$, can be achieved. Additionally, for a cavity $Q$ of 816 , an index modulation $(\delta n / n)$ as small as $1.3 \times 10^{-3}$ is sufficient for tuning cavity $\omega$ for on-off optical switching applications. ${ }^{5}$

In Fig. 4 we plot the cavity $Q$ factor (filled circles) on a logarithmic scale and cavity $\omega$ (filled squares) as a function of number of periods $(N=2,3,4)$. Whereas the observed $\omega$ varies by less than $1 \%$, the $Q$ value increases sharply as $N$ is increased from 2 to 4 . The observed $Q$ factors are then fitted to an exponential function, $Q_{t}=Q_{0}{ }^{*} \exp (\kappa N a)$, where $\kappa$ is a fitting parameter. The fit is good, and the deduced $\kappa$ is $0.90 \pm$ $0.05(\mu \mathrm{m})^{-1}$. Here, $\kappa$ is a measure of the light-trapping strength of the two photonic tunnel barriers that bound the cavity mode. A similar exponential dependence has been reported in the microwave range for an ideal 2D photonic crystal. ${ }^{9}$ This exponential dependence suggests that the observed increase in $Q$ is due to an increase in reflectivity of the photonic crystal mirror. It further suggests that $Q_{r}$ is significantly larger than $Q_{t}$, and a higher $Q$ value is achievable by an increase of $N$. The small variation in cavity $\omega$ may be due to the uncertainty in the sizes of the defect holes. To minimize such a variation, one must refine process control through better nanometer-scale fabrication.

In summary, a new microcavity design has been proposed and structures realized with a $2 \mathrm{D}$ photonic- crystal slab. The cavity $Q$-factor was measured to be $816 \pm 30$ for an $N=4$ cavity. Such a high- $Q$ cavity will permit realistic realization of spontaneous-emission modification and optical on-off switches.

The research at Sandia National Laboratories is supported by the U.S. Department of Energy. Sandia is a multiprogram laboratory operated by Sandia Corporation, a Lockheed Martin Company, for the U.S. Department of Energy under contract DE-AC04-94AL 85000. S. Y. Lin's e-mail address is slin@sandia.gov.

\section{References}

1. E. M. Purcell, Phys. Rev. 69, 681 (1946).

2. S. Haroche and D. Kleppner, Phys. Today $42(\mathbf{X}), 24$ (1989).

3. H. Yokoyama, Science 256, 66 (1992).

4. E. Yablonovitch, J. Opt. Soc. Am. B 10, 283 (1993).

5. P. R. Villeneuve, D. S. Abrams, S. Fan, and J. D. Joannopoulos, Opt. Lett. 21, 2017 (1996).

6. E. Ozbay, G. Tuttle, M. Sigalas, C. M. Soukoulis, and K. M. Ho, Phys. Rev. B 51, 13,961 (1998).

7. S. Y. Lin, J. G. Fleming, M. M. Sigalas, R. Biswas, and K. M. Ho, Phys. Rev. B 59, 15,579 (1999).

8. D. R. Smith, R. Dalichaouch, N. Kroll, S. Schultz, S. L. McCall, and P. M. Platzman, J. Opt. Soc. Am. B 10, 314 (1993).

9. S. Y. Lin, V. M. Hietala, S. K. Lyo, and A. Zaslavsky, Appl. Phys. Lett. 68, 3233 (1996).

10. D. Labilloy, H. Benisty, C. Weisbuch, T. F. Krauss, V. Bardinal, and U. Oesterle, Electron. Lett. 33, 1978 (1997).

11. J.-K. Hwang, H.-Y. Ryu, D.-S. Song, I.-Y. Han, H.-W. Song, H.-K. Park, and Y.-H. Lee, Appl. Phys. Lett. 76, 2982 (2000).

12. O. J. Painter, A. Husain, A. Scherer, J. D. O'Brien, I. Kim, and P. D. Dapkus, J. Lightwave Technol. 17, 2082 (1999). An estimate of the upper-bound $Q$ value of 600 has been reported as a result of photoluminescence measurements.

13. J. S. Foresi, P. R. Villeneuve, J. Ferrera, E. R. Thoen, G. Steinmeyer, S. Fan, J. D. Joannopoulos, L. C. Kimberling, H. I. Smith, and E. P. Ippen, Nature 390, 143 (1997).

14. In such a measurement, the luminescence transition linewidth must be deconvoluted to allow for a quantitative analysis of cavity linewidth and therefore of cavity $Q$.

15. S. Noda, A. Chutinan, and M. Imada, Nature 407, 608 (2000).

16. E. Chow, S. Y. Lin, S. G. Johnson, P. R. Villeneuve, J. D. Joannopoulos, J. R. Wendt, G. A. Vawter, W. Zubrzycki, H. Hou, and A. Alleman, Nature 407, 983 (2000).

17. S. G. Johnson, S. Fan, P. R. Villeneuve, J. D. Joannopoulos, and L. A. Kolodziejski, Phys. Rev. B 60, 5751 (1999).

18. P. R. Villeneuve, S. Fan, S. G. Johnson, and J. D. Joannopoulos, IEE Proc. Optoelectron. 145, 384 (1998).

19. For calculation of single-hole defects, the hole diameter is varied from $d^{\prime}=0.3$ to $1.0 \mathrm{a}$. For the supercavity, the hole diameter varies from $d^{\prime}=0.3$ to $0.5 a$.

20. S. Y. Lin and G. Arjavalingam, Opt. Lett. 18, 1666 (1993).

21. When other losses, such as dielectric loss and TE-or-TM mode conversion loss, become comparible to tunneling loss, this dependence is no longer valid. 